\title{
Comparison of Intraoperative Sonography and Histopathologic Evaluation of Tumor Thickness and Depth of Invasion in Oral Tongue Cancer: A Pilot Study
}

\author{
(D) B.C. Yoon, (DM.D. Bulbul, (D) P.M. Sadow, (D).C. Faquin, (D).D. Curtin, (DM.A. Varvares, and (D)A.F. Juliano
}

\begin{abstract}
BACKGROUND AND PURPOSE: For primary squamous cell carcinoma of the oral tongue, accurate assessment of tumor thickness and depth of invasion is critical for staging and operative management. Currently, typical imaging modalities used for preoperative staging are CT and MR imaging. Intraoperatively, CT or MR imaging cannot provide real-time guidance, and assessment by manual palpation is limited in precision. We investigated whether intraoperative sonography is a feasible technique for assessment of tumor thickness and depth of invasion and validated its accuracy by comparing it with histopathologic evaluation of the resected specimen.
\end{abstract}

MATERIALS AND METHODS: Twenty-six patients with squamous cell carcinoma of the oral tongue who underwent tumor resection by a single surgeon between March 31, 2016, and April 26, 2019, were prospectively identified. Intraoperative sonography was obtained in planes longitudinal and transverse to the long axis of the tumor. Twenty-two patients had archived images that allowed measurements of tumor thickness and depth of invasion sonographically. Two patients had dysplasia and were excluded. The remaining 20 patients had histologic tumor thickness and histologic depth of invasion measured by a single pathologist.

RESULTS: The mean sonographic tumor thickness was $7.5 \pm 3.5 \mathrm{~mm}$, and the mean histologic tumor thickness was $7.0 \pm 4.2 \mathrm{~mm}$. Mean sonographic depth of invasion and histologic depth of invasion were $6.6 \pm 3.4$ and $6.4 \pm 4.4 \mathrm{~mm}$, respectively. There was excellent correlation between sonographic and histologic measurements for both tumor thickness and depth of invasion with Pearson correlation coefficients of 0.95 (95\% Cl, 0.87-0.98) and 0.95 (95\% Cl, 0.87-0.98), respectively.

CONCLUSIONS: Intraoperative sonography can provide reliable, real-time assessment of the extent of tongue tumors.

ABBREVIATIONS: DOI = depth of invasion; OTSCC $=$ oral tongue squamous cell carcinoma; $\Pi$ = tumor thickness; uDOI = ultrasound-derived depth of invasion; US = sonography; uTT = ultrasound-derived tumor thickness

T he recently updated, eighth edition of the Cancer Staging Manual of the American Joint Committee on Cancer incorporates the depth of invasion (DOI) of oral cavity cancers as an independent prognostic factor, in addition to tumor thickness (TT). ${ }^{1}$ Preoperative CT and MR imaging have been shown to delineate TT and DOI with good correlation to pathologically determined TT and DOI for lesions of $>5 \mathrm{~mm} .{ }^{2,3}$ However, the ability to perform intraoperative real-time assessment of tumor thickness and DOI as well as deep margin extent remains limited. Currently, manual palpation is the mainstay technique for tumor

Received March 19, 2020; accepted after revision April 24.

From the Departments of Radiology (B.C.Y.), and Pathology (P.M.S., W.C.F.), Massachusetts General Hospital, Boston, Massachusetts, and Departments of Otolaryngology Head and Neck Surgery (M.D.B., M.A.V.), Radiology (H.D.C., A.F.J.), and Pathology (P.M.S., W.C.F.), Massachusetts Eye and Ear, Boston, Massachusetts.

Please address correspondence to A.F. Juliano, MD, Massachusetts Eye and Ear, 243 Charles St, Boston, MA 02114; e-mail: Amy_Juliano@meei.harvard.edu; @amyfjuliano

http://dx.doi.org/10.3174/ajnr.A6625 evaluation during an operation in attempting to achieve the goal of a gross $1-\mathrm{cm}$ clearance at all margins with a goal of $0.5-\mathrm{cm} \mathrm{mi-}$ croscopic clearance on permanently fixed tissue. ${ }^{4}$ Although manual palpation may be adequate for determination of the more superficial, mucosal extent of a tumor, it is limited for precise assessment of the deep margin and the border between involved tissue and normal/healthy tissue. Furthermore, the current criterion standard for TT and DOI is histologic evaluation, which can only be performed after the tumor has been resected.

Sonography (US) is being increasingly recognized as a valuable tool in the assessment of intraoral tumor extent. ${ }^{3,5}$ For instance, a recent study demonstrated that intraoperative sonography can be used for adequate demarcation of the deep-resection margins for tongue cancers. ${ }^{6}$ In this study, we extend the previous findings to determine the accuracy of intraoperative sonography in assessing TT (uTT) and DOI (uDOI) of oral tongue squamous cell carcinomas (OTSCC), comparing them with the criterion standard postexcision, histopathologically assessed TT and DOI. 


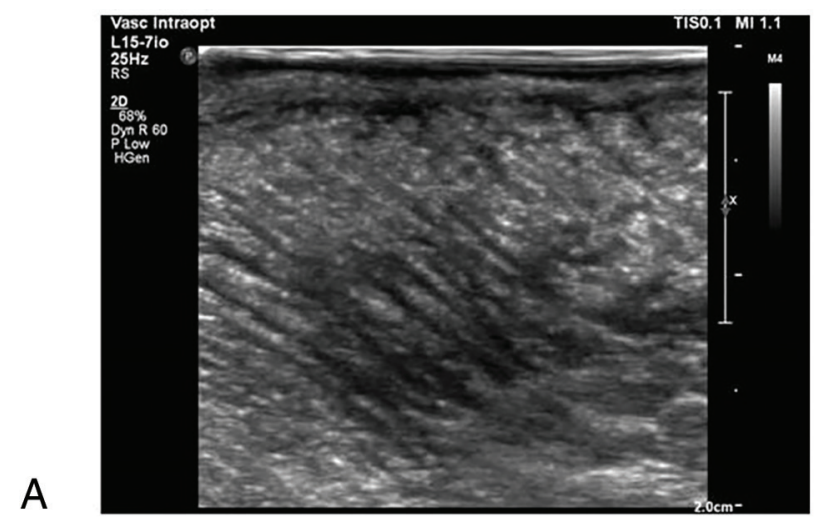

B
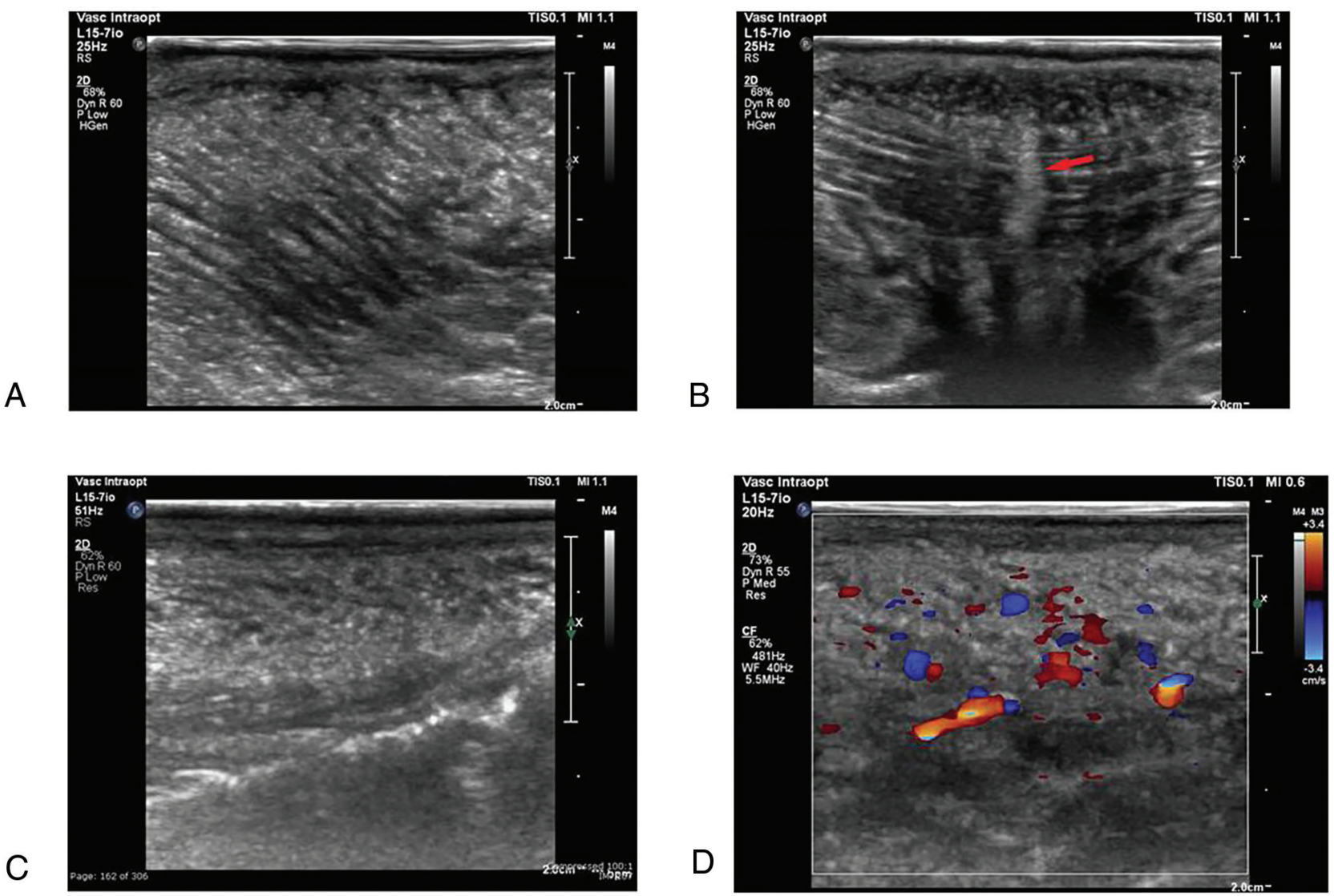

FIG 1. Gray-scale sonographic images of the normal oral tongue $(A-C)$. Normal muscle striations can be seen in the paramedian oral tongue ( $A$, sagittal) and in the midline dorsum of the tongue ( $B$, transverse). Note that in the midline, the median fibrous septum is discernable as an echogenic line (arrow in $B$ ). At the lateral edge of the oral tongue, the parenchyma is mildly heterogeneous and hyperechoic ( $C$, sagittal). $D$, Doppler sonography image of the lateral oral tongue shows fairly even distribution of blood flow throughout the parenchyma.

\section{MATERIALS AND METHODS Study Design}

Patients with previously untreated OTSCC were prospectively identified. They underwent tumor resection under US guidance by a single surgeon between March 31, 2016, and April 26, 2019. All US was performed by a single head and neck radiologist along with the surgeon and an US technologist. Patient data were obtained from medical records, including age, sex, and histologic tumor stage. This study was approved by the institutional review board of Massachusetts Eye and Ear.

\section{Surgical Resection with Intraoperative Sonographic \\ Guidance}

Each patient was placed under general anesthesia. Good exposure was gained of the oral cavity by gentle retraction of the lips and oral commissures. Visual inspection and manual palpation of the tongue tumor was performed to identify tumor location and estimate tumor extent.

Intraoperative US was performed before, during, and following tumor resection using a broadband compact linear array transducer (L15-7io; Philips Healthcare). The US transducer was sheathed with a sterile probe cover, with US gel placed inside the probe cover against the transducer end. Copious sonography gel was applied to the oral tongue on top of the tumor. The probe was then placed on the gel/tumor by the radiologist, and the tumor was imaged. Compared with the normal tongue (Fig 1), the tumor is abnormally hypoechoic and distorts the normal tongue architecture (Fig 2). Extra care was taken to rest the probe gently on the gel without applying undue pressure on the tumor, to prevent external forces from the probe compressing and thereby potentially distorting and artificially altering the measured uTT and uDOI (Fig 3). Real-time scanning of the tumor was performed in all directions, to gain an understanding of tumor size, extent, morphology, and contour both at the superficial and deep margins. Using B-mode and Doppler sonography, we obtained still images and cine video clips in planes longitudinal and transverse to the long axis of the tumor, with the transducer oriented perpendicular to the deepest portion of the tumor. Still images of representative landmark locations demonstrating uTT and uDOI were obtained.

Partial glossectomy then commenced, with the surgeon bearing in mind the attendant trajectory needed to produce approximately $1.0 \mathrm{~cm}$ of gross deep margin clearance. ${ }^{6}$ One-third to onehalf of the way through tumor extirpation, the surgeon paused and gray-scale and Doppler US was again performed to evaluate the deep margin of resection along with inspection and palpation (Fig $3 E$ ). The tumor and resection trajectory were noted, and the margin of clearance between the tumor and cut edge was noted and measured at various points along the trajectory.

Resection then resumed, with the surgeon modifying the resection trajectory as needed on the basis of the clearance margin 

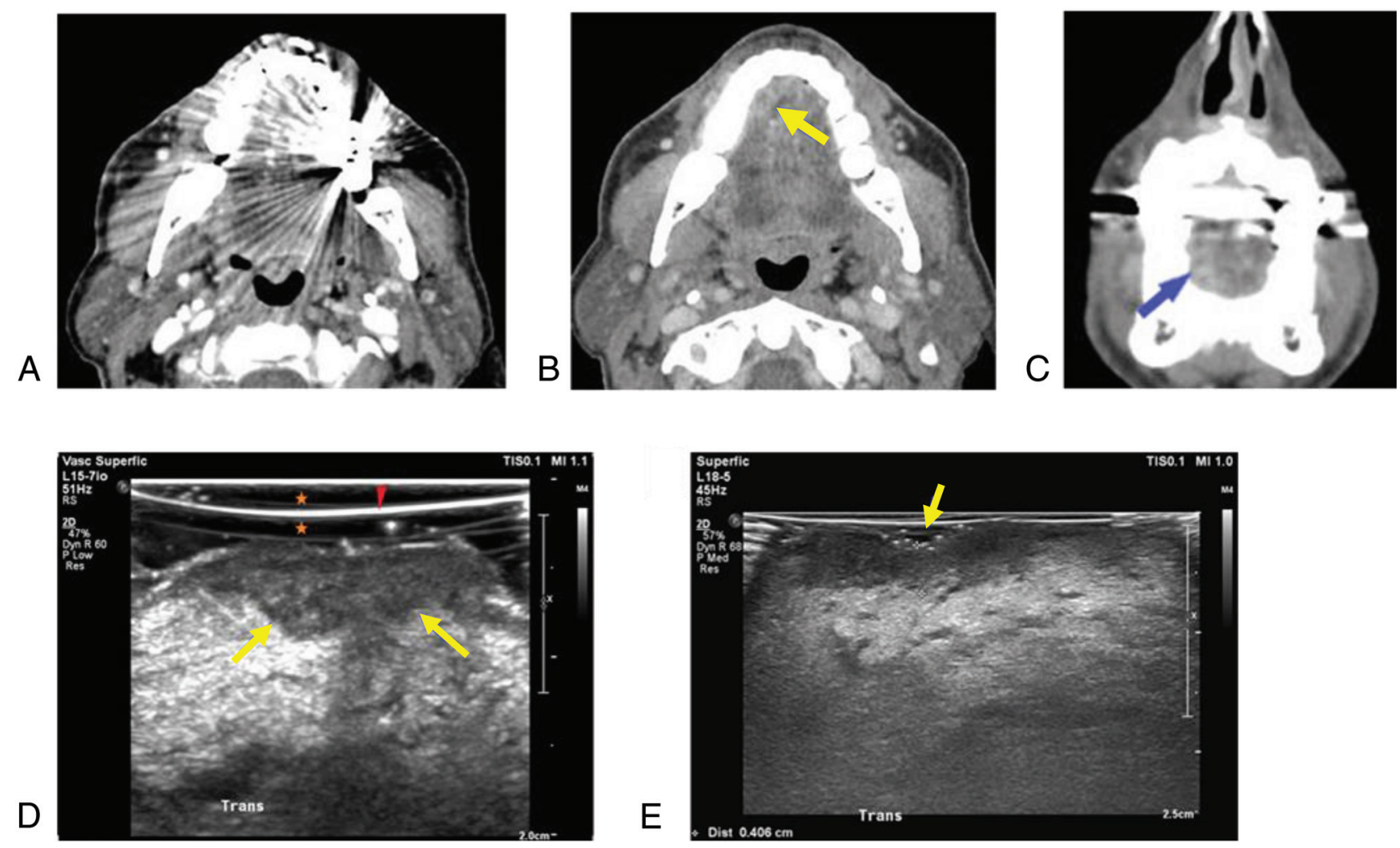

FIG 2. A, Axial contrast-enhanced CT image through the oral tongue is degraded by streak artifacts from metallic dental amalgam; the known right lateral oral tongue cancer cannot be appreciated. $B$, Re-angled contrast-enhanced CT image scanned at a tilted angle to avoid streak artifacts shows an area of subtle heterogeneous enhancement (arrow), likely representing the tumor. C, Coronal reformatted CT image shows the area (arrow) appearing relatively subtle and ill-defined. $D$, Intraoperative gray-scale sonography image shows an area of lobulated hypoechogenicity representing the tumor (arrows). The sonography probe was sheathed in a sterile probe cover (arrowhead), with sonography gel on either side of it (asterisks). Note how the probe was suspended lightly on the gel against the tongue to avoid distorting the natural contour of the tumor. E, Intraoperative gray-scale sonography image shows an endophytic ulcerated area of the tumor (marked by calipers). Sonography gel (arrow) coats the tongue in a thick layer, enabling visualization of the natural contour of the tumor.

noted. Following complete tumor extirpation, sonography was performed for a third time, this time on the resected surgical specimen (Fig 3F). Scanning in all directions was performed, cine video images were obtained in longitudinal and transverse planes, and still images were acquired at representative locations, with special attention paid to the interface between tumor tissue and healthy tissue and the margin of clearance between that interface and the resected surface. The surgical specimen was then taken to the frozen section laboratory and examined by both the surgeon and the attending pathologist (see below).

\section{Imaging Analysis}

Measurements of uTT and uDOI from intraoperative sonography images were made by 2 neuroradiologists, one with 15 years of neuro-/head and neck radiology experience (A.F.J.) and the other with 6 years of neuro-/head and neck radiology experience (B.C.Y.). Images were displayed on the PACS. The uTT was measured from the superficial surface of the tumor to the deepest point of tumor invasion (Fig 4), following a path that is perpendicular to the tongue surface. The uDOI was measured from the level of projected normal mucosal surface ${ }^{7}$ basement membrane adjacent to the tumor to the deepest point of tumor invasion (Fig 4), along a path perpendicular to the tongue surface. Consensus measurements were used for analysis.

\section{Statistical Analysis}

Statistical analysis was performed using GraphPad Prism software (Version 8; GraphPad Software). The Pearson correlation was used to determine the correlation between the sonographic and histopathologic assessments.

\section{Intraoperative and Postoperative Tissue Analysis}

Histopathologic analysis on the resected specimen was performed in 2 stages: measurements made by gross and microscopic evaluation intraoperatively, including selected frozen sections of the specimen, as is routine for surgery, and routine gross and histopathologic analyses postoperatively.

In the frozen section laboratory, the specimen was oriented by the surgeon along with the head and neck pathologist (P.M.S.). Gross measurements of the resection specimen were made along with gross measurements of the tumor to the closest surgical margins, leaving the oriented specimen largely intact for further prosection following fixation in formalin. After gross measurements of the tumor distance to the surgical margins (in centimeters or millimeters) were recorded, the closest margins to the tumor (on the main specimen), up to 6, were taken directly from the specimen, with a perpendicular (radial) section made to approximate the closest distance of tumor to the true surgical resection edge. This tissue was frozen in optimal cutting temperature compound 


\section{A}
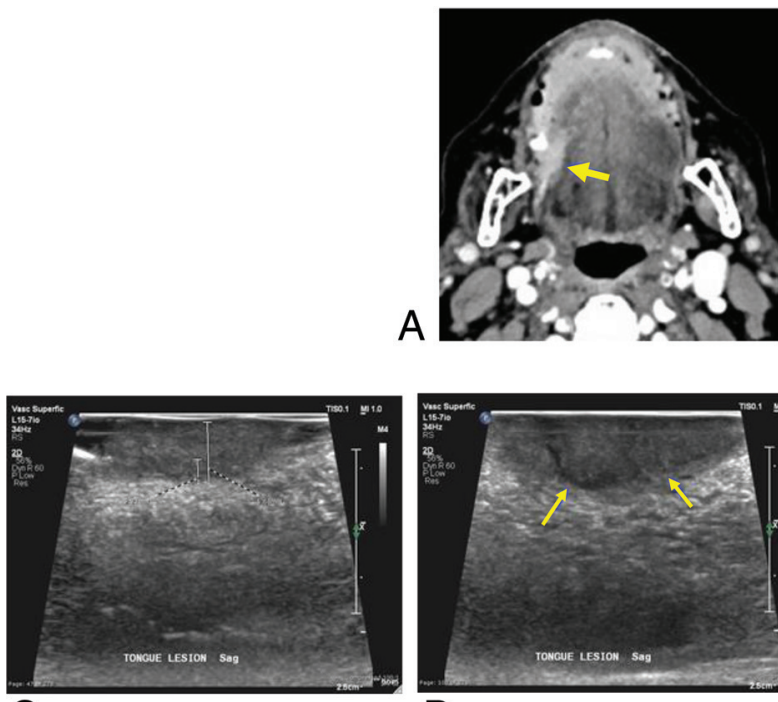

C

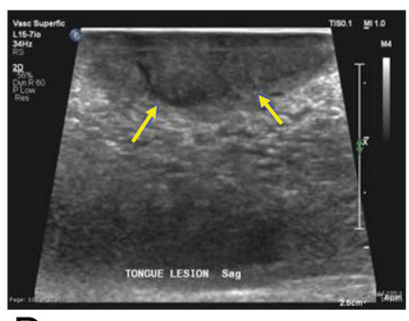

D
B

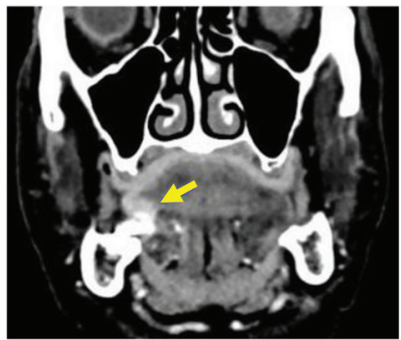

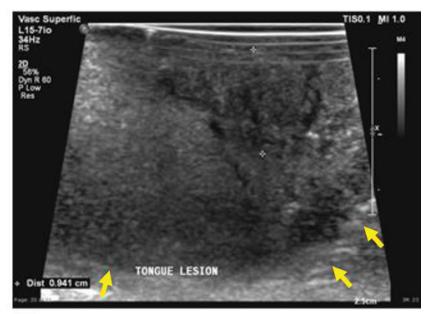

$\mathrm{F}$

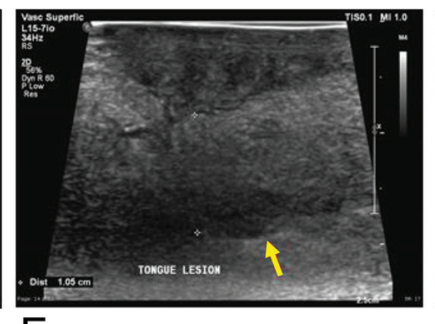

E

FIG 3. Axial $(A)$ and coronal $(B)$ contrast-enhanced $C T$ images show the enhancing right lateral tongue tumor (arrows in $A$ and $B$ ). However, it is difficult to appreciate on these images whether there may be exophytic or endophytic components that may render DOI different from TT. $C$, Intraoperative gray-scale sonography image obtained at the edge of the tumor demonstrates the exophytic nature of the tumor at that site. Rulers show measurements taken for uDOI (on the left) and uTT (on the right). D, The arrows denote the deep margin of the tumor. E, Intraoperative sonography image obtained during resection but before complete extirpation. Air can be seen at the resected interface as an echogenic line (arrow). Calipers show the margin of clearance between tumor and resection plane. F, Intraoperative sonography image obtained following complete extirpation of tumor. Air is seen throughout the resected edge (arrows). Calipers demarcate the tumor. Special attention was paid to ensure that the resection margins were free of tumor.

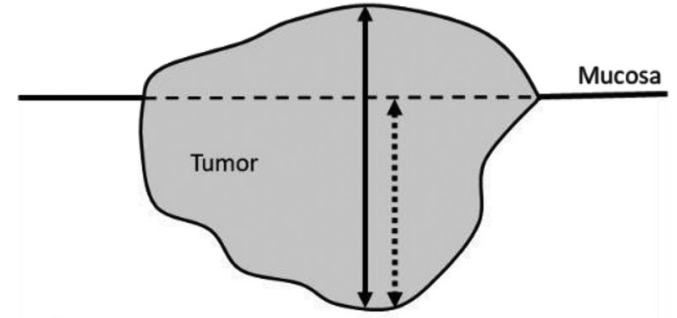

A
B

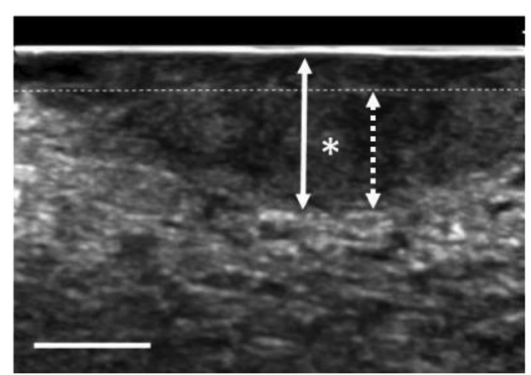

$\longleftrightarrow$ Tumor thickness

$4 . .$. Depth of invasion

FIG 4. $A$, Schematic representation of tumor thickness and depth of invasion. $B$, Representative, intraoperative sonography image of a tongue squamous cell carcinoma. The lesion is seen as a circumscribed, hypoechoic mass (asterisk). Scale bar $=1 \mathrm{~cm}$.

with a $4-\mu \mathrm{m}$ section cut using a cryostat. The tissue section, placed on positively charged glass slides, was fixed in ethanol and stained with hematoxylin-eosin for intraoperative histologic review. Distance to the surgical margins was measured microscopically using a professional light microscope (Olympus BX51 microscope with Olympus DP27 camera). Following intraoperative evaluation of surgical margins, the tissue (main specimen) was placed in formalin preservative and fixed for permanent processing and histologic review. The frozen "margin" remnant tissue was transferred to cassettes for formalin fixation (the optimal cutting temperature compound is water/formalin soluble) and re-analyzed on permanent section following proper fixation and paraffin embedding.

\section{RESULTS}

A total of 26 patients with OTSCC were included in this series. Among this cohort, 22 patients had archived images in the PACS that allowed measurements to be made sonographically (uTT,
uDOI). Two patients had dysplasia and were excluded. Of the remaining 20 patients, all had both histopathologic TT and DOI measured by a single pathologist (Table).

The mean uTT was $7.5 \pm 3.5 \mathrm{~mm}$, and the mean histopathologic TT was $7.0 \pm 4.2 \mathrm{~mm}$. The mean $\mathrm{uDOI}$ and histopathologic DOI were $6.6 \pm 3.4 \mathrm{~mm}$ and $6.4 \pm 4.4 \mathrm{~mm}$, respectively. There was a strong correlation between sonographic and histologic measurements for both TT and DOI with Pearson correlation coefficients of 0.95 (95\% CI, 0.87-0.98) and 0.95 (95\% CI, 0.870.98), respectively (Fig 5).

\section{DISCUSSION}

Sonographic imaging of the tongue tumor, surrounding tissues, and interface between tumor and normal tissue was successfully performed intraoperatively in all cases without adverse events. A strong correlation was observed between sonographic and histopathologic measurements of both TT and DOI. 


\begin{tabular}{|c|c|c|c|c|c|c|c|c|c|}
\hline Patient No. & Age (yr) & Sex & $\begin{array}{c}\text { uTT } \\
(\mathrm{mm})\end{array}$ & $\begin{array}{l}\text { hTT } \\
(\mathrm{mm})\end{array}$ & $\begin{array}{l}\text { uDOI } \\
(\mathrm{mm})\end{array}$ & $\begin{array}{l}\text { hDOI } \\
(\mathrm{mm})\end{array}$ & $\begin{array}{l}\text { Lymphovascular } \\
\text { Invasion }\end{array}$ & $\begin{array}{l}\text { Perineural } \\
\text { Invasion }\end{array}$ & $\begin{array}{c}\text { Nodal } \\
\text { Metastasis }\end{array}$ \\
\hline 1 & 75 & $\mathrm{~F}$ & 5 & 5 & 5 & 5 & No & No & No \\
\hline 2 & 70 & $\mathrm{~F}$ & 9 & 9 & 9 & 9 & No & Yes & No \\
\hline 3 & 44 & $M$ & 4 & 5 & 4 & 3 & No & No & No \\
\hline 4 & 81 & $M$ & 7 & 5 & 7 & 5 & No & No & No \\
\hline 5 & 66 & $M$ & 4 & 2.5 & 3 & 2.5 & No & No & NA \\
\hline 6 & 29 & $\mathrm{~F}$ & 6.5 & 4 & 6.5 & 4 & No & No & NA \\
\hline 7 & 53 & $\mathrm{~F}$ & 7 & 6 & 3 & 2 & No & Yes & No \\
\hline 8 & 27 & $M$ & 8 & 9 & 8 & 9 & Indeterminate & Yes & No \\
\hline 9 & 75 & $\mathrm{~F}$ & 3 & 2 & 3 & 1 & No & No & NA \\
\hline 10 & 61 & $M$ & 5 & 4 & 3 & 4 & No & No & No \\
\hline 11 & 61 & $M$ & 10 & 10 & 9 & 7 & No & No & No \\
\hline 12 & 46 & $M$ & 15 & 13 & 10 & 13 & No & Yes & No \\
\hline 13 & 46 & $M$ & 8.5 & 10 & 8.5 & 9 & No & Yes & Yes \\
\hline 14 & 65 & $M$ & 8 & 7 & 8 & 7 & No & Yes & No \\
\hline 15 & 57 & $\mathrm{M}$ & 14 & 15 & 14 & 15 & No & No & Yes \\
\hline 16 & 65 & $M$ & 3 & 2 & 3 & 1.5 & No & No & No \\
\hline 17 & 76 & $\mathrm{~F}$ & 8 & 8 & 6 & 8 & No & No & No \\
\hline 18 & 78 & $M$ & 13 & 16 & 13 & 16 & Yes & Yes & No \\
\hline 19 & 82 & $M$ & 7 & 5 & 6 & 5 & No & Yes & No \\
\hline 20 & 50 & $\mathrm{~F}$ & 4 & 3 & 3 & 2.5 & No & No & NA \\
\hline
\end{tabular}

Note:-hTT indicates histologic tumor thickness; hDOI, histologic depth of invasion; NA, not applicable.

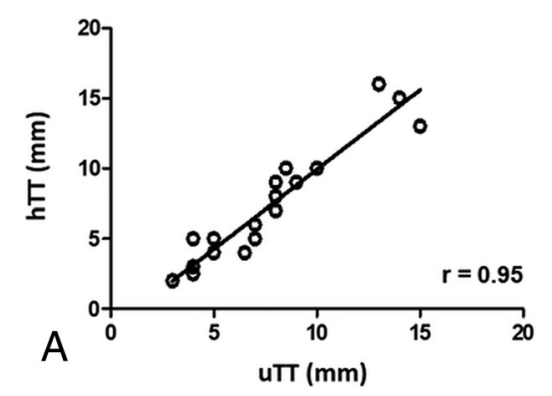

FIG 5. $A$, Correlation between sonography and histologic tumor thickness measurements $(r=$ Pearson coefficient; $n=20)$. $B$, Correlation between sonography and histologic depth of invasion measurements $(r=$ Pearson coefficient; $n=20)$.

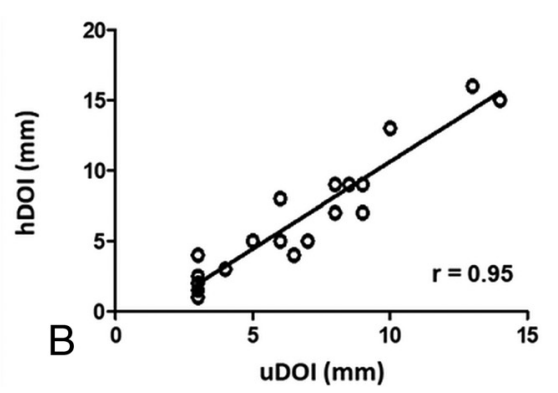

operators. In this series, for instance, the initial several cases were performed with the transducer placed directly over and applying pressure to the tumor, which effectively compresses the lesion and possibly underestimates the TT. With increasing experience and awareness of this issue and with the need to ensure that we distinguish exophytic from endophytic portions of the tumor, we began to apply a thick layer of sonography gel on the tumor and suspend the transducer within the gel without pressure on the tumor, thus avoiding distortion

Intraoperative US has been shown to be critically helpful in the guidance of tumor resection in various parts of the body. ${ }^{8}$ Realtime sonography performed immediately before and during surgical resection can help to inform the resection trajectory by demonstrating the tumor and illustrating exophytic or endophytic components, as well as the surrounding uninvolved tissue, clearance margin between tumor and resection surface, vessels, and so forth. With real-time sonography, the surgeon can be reassured that the resection trajectory (for the purpose of tumor clearance) is adequate and optimal, or can be alerted to the need to modify the resection trajectory (for the purpose of organ preservation) to balance the need for adequate tumor margins and preservation of the maximal amount of uninvolved tissue. This assurance is particularly critical for a small-but-functionally-critical organ such as the oral tongue, vital for speech, swallowing, food intake, and airway preservation. In this study, we have shown that sonography has good accuracy in determining the TT and DOI of oral tongue masses, ensuring that it is a reliable method for surgical guidance.

One of the limitations of sonography is the variability in image acquisition between different lesions and different of tumor configuration. Of note, histopathologic assessment can also be affected by distortion during resection and histologic preparation, including, among other mechanical distortions, tissue-retraction artifacts.

No substantial increase in operative time was observed in this series, though there remains a potential for prolonging the operative time depending on the availability of equipment and/or expertise of the sonographer at each facility/institution. Although the tumor margins were relatively well-defined in the current series, they may be more obscured depending on the tumor histology and/or transducer types. In addition, if the tumor shape is irregular, then measurements may be subject to variability depending on which point and path is selected for measuring purposes; however, this is the case for both sonography and frozen section evaluation.

\section{CONCLUSIONS}

This pilot study demonstrates that intraoperative sonography can provide objective, real-time assessment of TT and DOI with excellent correlation with histologic evaluation. This information is extremely helpful intraoperatively, allowing (1) resection with clear 
margins upfront, important since positive margins cannot be revised in second surgeries to a level of disease control of that of an initially negative resection margin; ${ }^{9-11}$ (2) choice of elective neck dissection to be performed in a clinically N0 neck (advisable at a threshold of $4 \mathrm{~mm} \mathrm{DOI}$ ) in the same surgical episode. It also provides accurate staging information for multidisciplinary management of the patient going forward. Additional work is needed to confirm the high level of correlation between sonographic and histologic measurements of TT and DOI and to deploy the use of ultrasound more widely in the care of patients with oral cancer.

Disclosures: William C. Faquin-UNRELATED: Board Membership: American Cancer Society, Comments: Editor-in-Chief for Cancer Cytopathology; Expert Testimony: medicolegal firms, Comments: rare expert testimony for medicolegal cases unrelated to this article.

\section{REFERENCES}

1. Lydiatt WM, Patel SG, O'Sullivan B, et al. Head and neck cancers: major changes in the American Joint Committee on Cancer eighth edition Cancer Staging Manual. CA Cancer J Clin 2017;67:122-37 CrossRef Medline

2. Weimar EA, Huang SH, Lu L, et al. Radiologic-pathologic correlation of tumor thickness and its prognostic importance in squamous cell carcinoma of the oral cavity: implications for the eighth edition Tumor, Node, Metastasis Classification. AJNR Am J Neuroradiol 2018;39:1896-902 CrossRef Medline
3. Shintani S, Yoshihama Y, Ueyama Y, et al. The usefulness of intraoral ultrasonography in the evaluation of oral cancer. Int J Oral Maxillofac Surg 2001;30:139-43 CrossRef Medline

4. Wong LS, McMahon J, Devine J, et al. Influence of close resection margins on local recurrence and disease-specific survival in oral and oropharyngeal carcinoma. Br J Oral Maxillofac Surg 2012;50:102-08 CrossRef

5. Narayana HM, Panda NK, Mann SB, et al. Ultrasound versus physical examination in staging carcinoma of the mobile tongue. $J$ Laryngol Otol 1996;110:43-47 CrossRef Medline

6. Tarabichi $\mathrm{O}$, Kanumuri V, Juliano AF, et al. Intraoperative ultrasound in oral tongue cancer resection: feasibility study and early outcomes. Otolaryngol Head Neck Surg 2018;158:645-48 CrossRef Medline

7. Hegde P, Roy S, Shetty $\mathrm{T}$, et al. Tumor infiltration depth as a prognostic parameter for nodal metastasis in oral squamous cell carcinoma. Int J Appl Basic Med Res 2017;7:252-57 CrossRef Medline

8. Grondona P, Meola C, Floris F, et al. Ultrasound-guided liver resection: early experience in a district general hospital. J Ultrasound 2008;11:162-67 CrossRef Medline

9. Patel RS, Goldstein DP, Guillemaud J, et al. Impact of positive frozen section microscopic tumor cut-through revised to negative on oral carcinoma control and survival rates. Head Neck 2010;32:1444-51 CrossRef Medline

10. Bulbul MG, Tarabichi O, Sethi RK, et al. Does clearance of positive margins improve local control in oral cavity cancer? A meta-analysis. Otolaryngol Head Neck Surg 2019;161:235-44 CrossRef Medline

11. Varvares MA, Poti S, Kenyon B, et al. Surgical margins and primary site resection in achieving local control in oral cancer resections. Laryngoscope 2015;125:2298-307 CrossRef Medline 